\title{
Study of the robustness of the European air routes network
}

\author{
Oriol Lordan $^{1}$, Joel Florido ${ }^{1}$, Jose M. Sallan ${ }^{1}$, Vicenc Fernandez ${ }^{1}$, Pep \\ Simo $^{1}$ and David Gonzalez-Prieto ${ }^{2}$
}

\author{
${ }^{1}$ Department of Management, Universitat Politècnica de Catalunya. BarcelonaTech, \\ Terrassa, Spain, 08222. \\ ${ }^{2}$ Volotea. Travessera de Gràcia, 56. Barcelona, Spain, 08006. \\ oriol.lordan@upc.edu; joel.florido@estudiant.upc.edu; jose.maria.sallan@upc.edu; \\ vicenc.fernandez@upc.edu; pep.simo@upc.edu; david.gonzalez@,volotea.com
}

\begin{abstract}
In this working paper we make analysis on flight flow network structure of Europe. The aim of this study is to analyze the robustness of the European civil European air transportation network from the perspective of the theory of complex networks. This study is based on analyzing the behavior of the network when its nodes (airports) are removed by random attacks and deliberate attacks. The selection of nodes on deliberate attacks is based on four criteria: degree, betweenness, pagerank and eigenvector. This study aims to contribute to the understanding of the logistics network of European commercial flights, analyzing their strengths and weaknesses. The calculation of network statistical indicators as degree and betweenness distribution reveals that the flight flow network has the small-world characteristics and scale-free property.
\end{abstract}

Keywords Robustness • Network • Air Routes

\section{Introduction}

Air transport is a real revolution in any socioeconomic area, bringing commercial activity between people and enabling social relations. Currently, air transport is directly responsible of globalization, allowing a cultural, economic and political development.

Many systems are made by a large number of highly interconnected dynamical units. The first approach to capture the global properties of such systems is to model them as graphs whose nodes represent the dynamical units and the links stand the interactions between two dynamical units. A central issue in the analysis of complex networks is the assessment of their security and stability. The main aim is to understand, predict, and possibly even control the behaviour of a networked system under attacks of dysfunctions of any type.

Air traffic is on dynamic environment, where airports and routes may be temporarily closed for reasons unrelated to performance and functionality. The closure of airports for environmental causes, accidents, security alerts, strikes in the sector and terrorist attacks produces high costs for the airline industry.

The robustness allows us to analyze the effects of random attacks as weather inclemency or deliberate attacks as terrorist attacks on the air transportation 
network These attacks have effect on airlines, regions or airspaces. The study of the robustness evaluates the ability of a network to avoid a malfunction when a fraction of its components is damaged [1]. Thus, we can analyze the resistance and tolerance to deliberate attacks and/or random attacks on the network.

In recent years, the European air traffic has been affected by this type of incidents that caused the cancellation of flights and closure of some of the largest airports in terms of traffic. Recent examples are found on IATA Economics Briefing. The impact of hurricane Sandy (IATA Economics Briefing. The impact of hurricane Sandy) on the east coast of the U.S., produced cancellation of 17,000 flights and an economic impact of US\$500 M. The ash plume from Iceland's Eyjafjallajokull (IATA Economics Briefing. The impact of Eyjafallajokull's volcanic ash plume) volcano led to the progressive closure of much of Europe's airspace over a period of seven days, causing over 100,000 flights to be cancelled [2],[3].

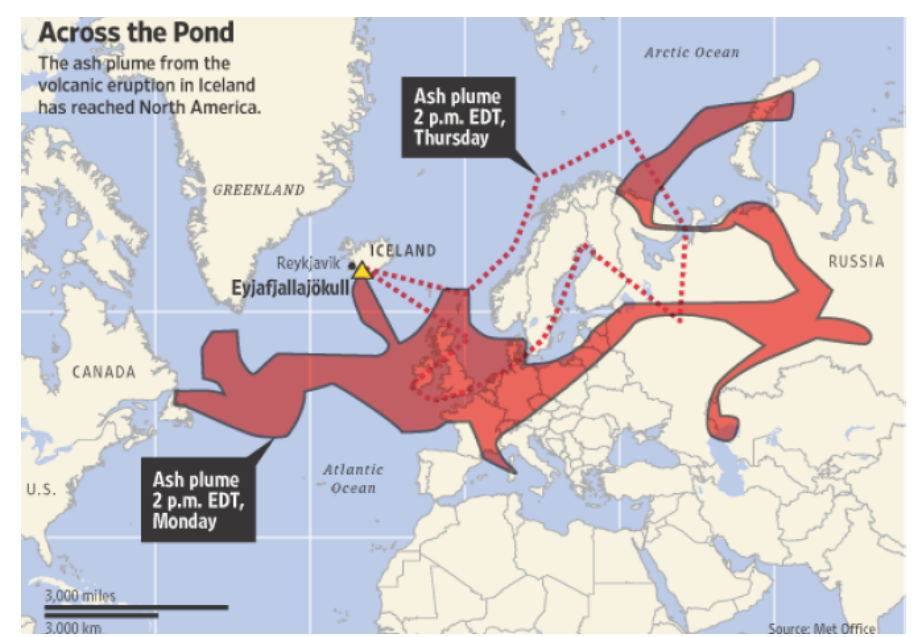

Fig. 1. The spread and shift of the plume. Source: The Economist, UK Met Office

In the last years have been published studies of complex networks which are in the real world (eg, [4],[5],[6],[7]), constituting one of scientific basis to analyze European network.

The aim of this study is to analyze the robustness of the network of European civil air transport routes from the perspective of the theory of complex networks. This study is based on analyzing the behavior of the network when its nodes (airports) are removed by random attacks and deliberate attacks. The selection of nodes on deliberate attacks is based on four criteria: degree, betweenness, pagerank and eigenvector.

\section{Methodology and sample}

The European air network can be formally described in terms of a graph $G(X, E)$. Here $X=\left\{X_{i}\right\}$ indicates the set of $X$ nodes (airports). These nodes can be connected and $E=\left\{e_{i j}\right\}$ indicates the set of actual links (routes) between pair of nodes.

The European air network contains 661 nodes and 8,104 links. It has taken into account all the links between airports which had at least one flight. In this study, we only considered the flights between European airports. We have not taken into account intercontinental routes.

The data of this study are obtained from the database Schedule Reference Service (SRS) Analyzer of IATA. The SRS Analyser is an online query tool designed to access SRS data, for market analysis and research. It brings worldwide airline passenger 
schedule information from the SRS. Following the theory of networks, we have done data processing for analysis of the network topology and robustness. This analysis was implemented and programmed by the language R. More specifically, for analyzing graphs and complex networks we have used the 'igraph' library.

The robustness is measured through the size of the giant component. Giant component of a graph is the largest subgraph that is still connected. We study the changes in giant component when a small fraction of the nodes is removed. In order to describe the concept of robustness of a network, one has first to discuss the issue of network malfunctioning. This may be done under two different points of view:

1. By considering the deactivation (deletion) of a node, together with all the links having such a node as an extreme.

2. By considering the deactivation (deletion) of one or several edges, without deleting any node.

Because the flexibility of the edges in the network is large, we only consider the attack on the network nodes, the attack on airports. We use two methods to attack the network. Attack methods can be divided into random attacks and deliberate attacks. By error or failure we mean the removal of randomly selected nodes. This test was performed 1,000 times and then we calculated the average of the Giant Component based on the removed nodes. Instead, we call attack the targeted removal of the most important nodes. We will consider four different criteria to determine the importance of a node: degree, betweenness, pagerank and eigenvector.

\section{Results}

The basic statistical measures are normally the average node degree $(<K>)$, the diameter (D), the average path length (L), the efficiency (E) and the clustering coefficient (C). In the Table 1, we can see the characteristics of the European flight flow network.

\begin{tabular}{|c|c|c|c|c|c|c|}
\hline Nodes & Edges & <k> & D & L & E & C \\
\hline 661 & 8104 & 24.520 & 7 & 2.7121 & 0.406 & 0.545 \\
\hline
\end{tabular}

Table 1. Characteristics of the European flight flow network

The small-world property in real networks is often associated with the presence of clustering, denoted by high values of the clustering coefficient. Therefore, we can consider that European flight flow is small-world network because has both a small value of $L$ and high clustering coefficient.

In order to gain greater insight into the structure and evolution of European air transportation network, we calculate the degree distribution of the airports. The degree distribution of an airport is the number of other airports to which is connected by a nonstop flight. The data suggest that most if the real networks display power law shaped degree distribution with exponents varying in the range $(2<\gamma<3)$. When the size of the system is small and the degree distribution $\mathbf{P}(k)$ is heavy-tailed, it is advisable to measure the cumulative degree distribution $P_{\text {cum }}(k)$. Consequently, if $\mathrm{P}(\mathrm{k}) \sim \mathrm{A} k^{-\gamma}$, the exponent $\gamma$ can be obtained from $\mathrm{P}_{\text {cum }}(\mathrm{k})$ as one plus the slope of $P_{\text {cum }}(k)$ in a $\log -\log$ plot. $\gamma=1+\gamma_{\text {cum. }}$. In the double logarithmic coordinate, the power-law distribution is a line with a negative slope, which is the main basis for judging whether the network obeys power-law distribution. The cumulative degree distribution and cumulative betweenness distribution of the European flight flow network is shown in Figure 2 and 3, respectively. In both cases, 
the line decays according to a power-law distribution, constituting a scale-free network.

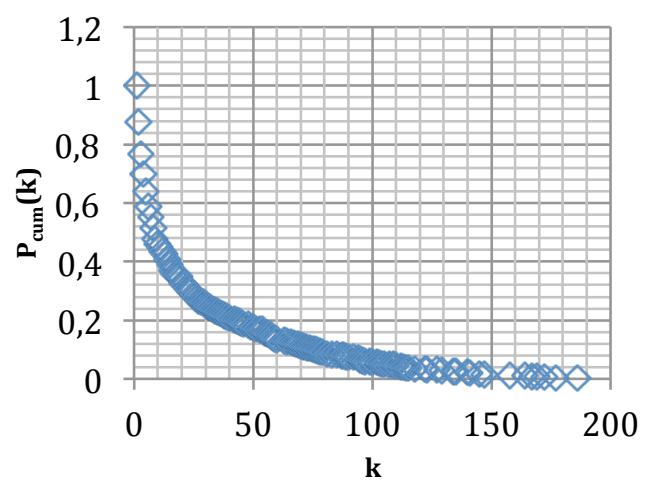

Fig. 2. Cumulative degree distribution

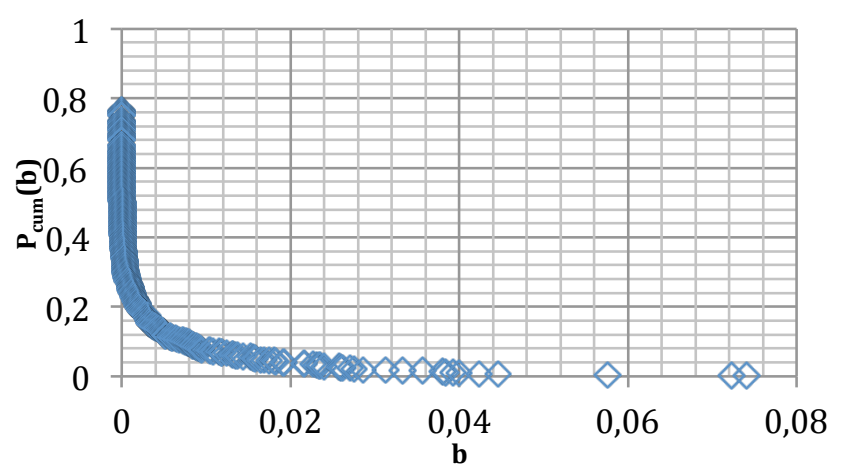

Fig. 3. Cumulative betweenness distribution

In the original coordinate, the degree distribution lines present a long tail characteristic. This illustrates that there are only a few high degree nodes in the network (hub airports) and most of the nodes have a low node degree (regional airports). The betweenness distribution lines present a long tail characteristic. This illustrates that there are only a few high betweenness node in the network and most of the nodes have a very low betweenness. Such a network fits the scale-free property.

Figures 4 and 5 show the cumulative degree distribution and cumulative betweenness distribution in double logarithm, respectively.

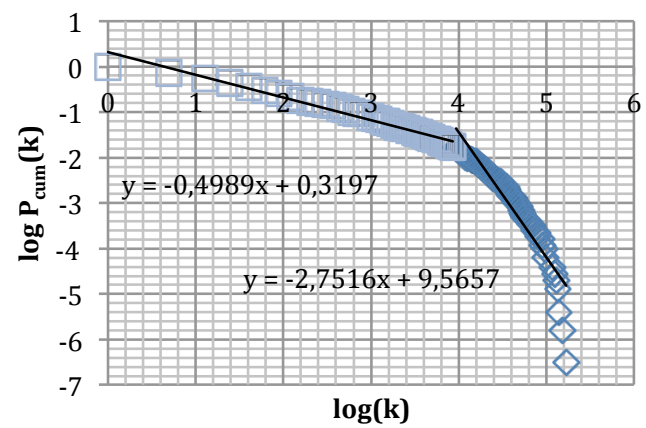

Fig. 4. Cumulative degree distribution in double logarithm

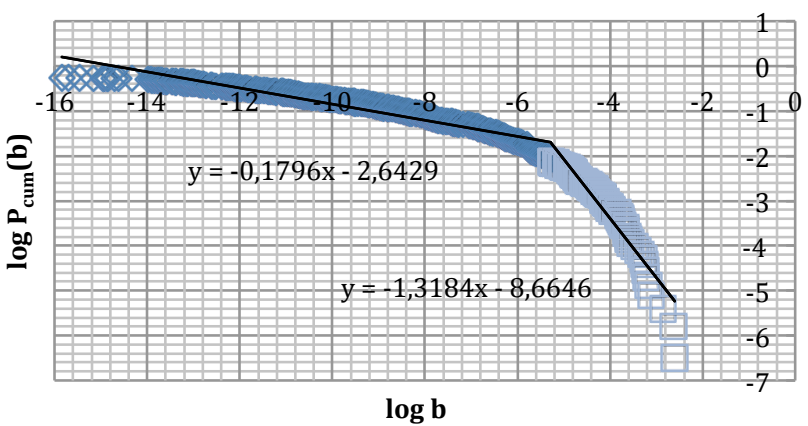

Fig. 5. Cumulative betweenness distribution in double logarithm

We can observe that in the double logarithmic coordinate (Figure 4), the degree distributions are almost negative slope lines, both in the first and second stages of two-step-fitting scheme, demonstrating that the network is a scale-free network. The slopes are $-0,4989$ and $-2,7516$. The exponent in the two stages is: $\gamma_{1}=1,4989$ and $\gamma_{2}=3,7516$.

We can observe that in the double logarithmic coordinate (Figure 5), the betweenness distributions are almost negative slope lines, both in the first and second stages of two-step-fitting scheme, demonstrating that the network is a scalefree network. The slopes are $-0,1796$ and $-1,3184$. The exponent in the two stages is: $\gamma_{1}=1,1796$ and $\gamma_{2}=3,3184$.

Secondly, Figure 6 shows the comparative results of the European network robustness according to the strategy (ie, random attacks and deliberate attacks). 


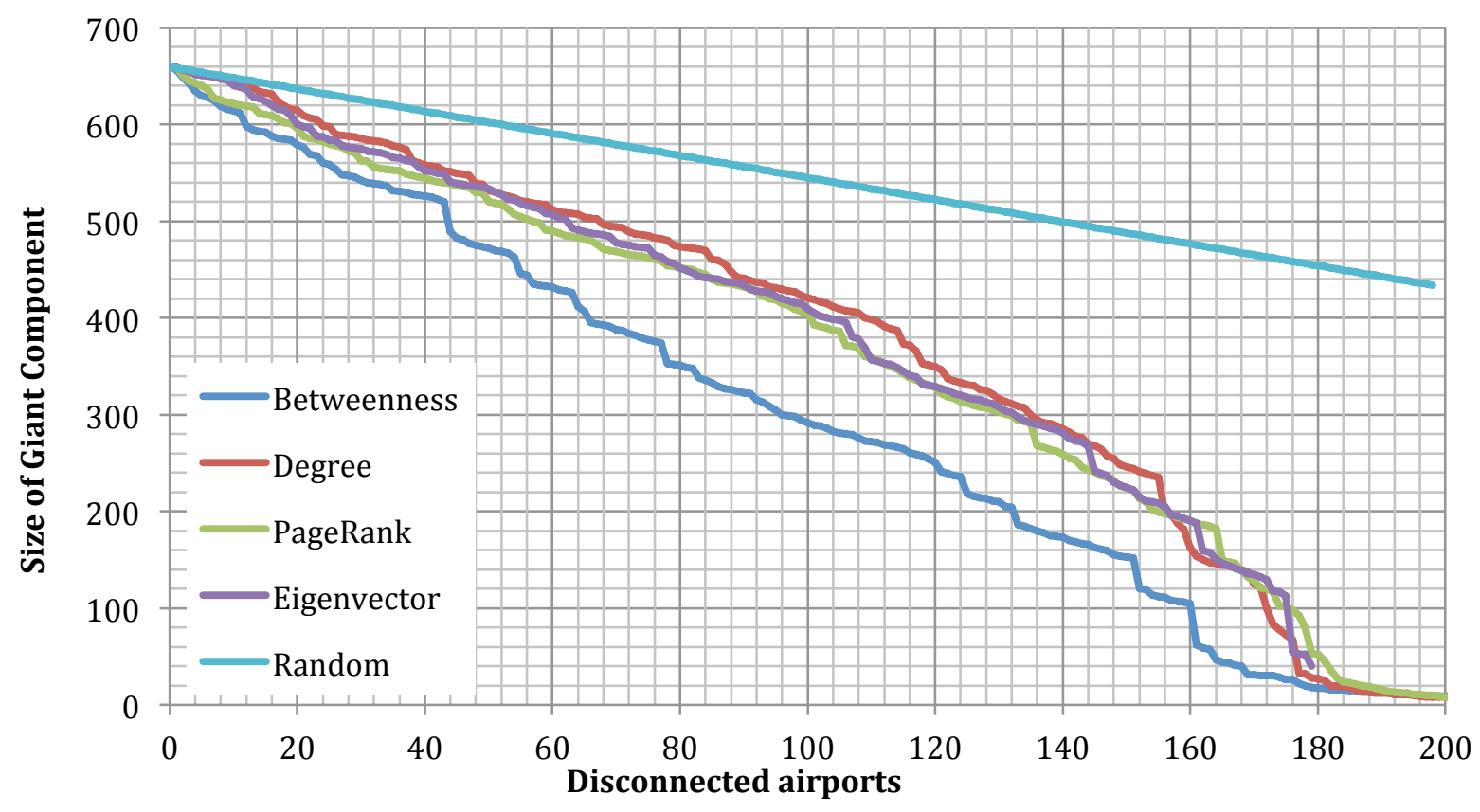

Fig. 6. Comparative performance of several node isolation criteria

As we can see in the results, deletion of nodes according to their betweenness centrality is the most efficient strategy to disintegrate the European air transportation network.

\section{Conclusions}

In this paper we make an analysis of the structure of the network of European air transport routes. We have used complex network theory and have obtained a flight flow model of Europe. The study allows us to know a number of significant results. The European air transport network is a "small world network" in which the number of nonstop connections in a particular city and the number of shortest paths that pass through a given city have distributions that are scale-free. Surprisingly, most connected nodes are not always the most central.

Furthermore, this analysis of the structure of the air transport network is important for two additional reasons. First, it allows us to identify the most efficient ways to design the network structure. Specifically, we can identify which airports are poorly connected and ways to minimize that problem. Secondly, hub airports play an important role in the disintegration of the network. It has been shown that this airports due to high betweenness keep the network connected and if there are failures on them it can be serious consequence on the network.

In order to analyze network survivability was used indicator of the maximum connected subgraph size (giant component). A comparison of the various attacks, the network drops faster when we eliminate nodes based on betweenness, which means that the damage is greater under betweenness. When approximately 160 nodes have been removed from the network, it is disconnected completely. However, when removed randomly, the network drops more slowly. This means that the development of the network depends on a few flights airports. When major airports are affected by natural disasters or failures, the entire network would be severely affected. Therefore, airports with high betweenness must ensure that operate safely and efficiently. Meanwhile, it should optimize the organization of airlines, improving multi -hub systems, and improve reliability and connectivity of the European network. 
Finally we believe that another interesting aspect is the study of the dynamic robustness. However, reorganizing the route map after the closure of one airport is not an easy action because of the number of agents that implies in terms of regulation, airport permissions, and air traffic redistribution.

\section{References}

1. Boccaletti, S., Latora, V., Moreno, Y., Chavez, M., Hwang, D.-U. (2006) Complex Networks: Structure and Dynamics. Physics Report, 424(4-5):75-308

2. Brooker, P. (2010) Fear in handful of dust: Aviation and the Icelandic volcano. Significance, 7(3):112-115.

3. Mazzocchi, M., Hansstein, F., Ragona, M., et al. (2010) The 2010 volcanic ash cloud and its financial impact on the European airline industry. In: ESifo Forum, Vol. 11: 92-100. Ifo Institute for Economic Research at the University of Munich

4. Guimera, R., Amaral, L.A.N. (2004) Modeling the world-wide airport network. The European Physical Journal B-Condensed Matter and Complex Systems, 38(2):381385

5. Xu, Z., Harriss, R. (2008) Exploring the structure of the U.S. intercity passenger air transportation network: A weighted complex network approach. GeoJournal 73:87-102.

6. Dang, Y., Ding, F., Gao, F. (2012) Empirical analysis on flight flow network survivability of China. Journal of Transportation Systems Engineering and Information, 12(6):177-185.

7. Guida, M., Maria, F. (2007) Topology of the Italian airport network: A scale-free small-world network with a fractal structure. Chaos, Solitons \& Fractals, 31(3):527536. 